\title{
Fuzzy Control System Using Nonlinear Friction Observer for the Mobile Robot
}

\author{
Woo-Young Lee ${ }^{1}$, Il-Seon Lim² ${ }^{2}$ Uk-Youl Huh ${ }^{3}$ \\ ${ }^{1}$ Dept. of Electrical Engineering, Inha University, Incheon 402-751, Korea \\ woollung@hotmail.com \\ ${ }^{2}$ Dept. of Electrical Engineering, Inha University, Incheon 402-751, Korea \\ islim@kopo.ac.kr \\ ${ }^{3}$ Dept. of Electrical Engineering, Inha University, Incheon 402-751, Korea \\ uyhuh@inha.ac.kr
}

\begin{abstract}
In this paper, a fuzzy control system with the nonlinear friction observer is presented. This observer is used to compensate the nonlinear friction that makes the position accuracy in a wheeled mobile robot be limited. Adding the nonlinear friction observer in the system, the tracking accuracy of the robot is improved without any breakaway when it traces the reference trajectory. And experimental results show that the fuzzy controller with the observer has better performance even though it has difference between the right wheel inertia and the left wheel inertia.
\end{abstract}

\section{Introduction}

Fuzzy logic control is based on human intelligence and an expert's knowledge and performs well for the system that has nonlinear characteristic. And fuzzy logic control is robust on the change of system parameters and copes with the disturbance that has bad effects on the system [1][2]. Because of these merits, fuzzy logic control is used to control a mobile robot nowadays. A mobile robot uses the sensor information and makes path planning. After that, it carries out path tracking. In the process, the path consists of straight routines and curves. However, due to contour error and direction error, the robot stays easily out of the way. Those errors are caused by some unknown disturbance like nonlinear friction disturbance. In mobile robot, nonlinear friction is the factor which makes stick-slip induced by stiction and Stribeck effect [3]. To reduce these errors, we add a nonlinear friction observer to fuzzy controller. It is necessary to control the robot with high performance and accuracy by proper modeling and friction compensation. In designing the fuzzy controller with an observer, we separate friction torque characteristic into two parts as a positive part and a negative part due to nonlinear characteristic. According to analysis using this observer, we can compensate the errors with the observer that may cause breakaway when the robot moves. Simulation results show that fuzzy controller using the observer has good performance even if the robot has different inertia between two wheels.

P.M.A. Sloot et al. (Eds.): ICCS 2002, LNCS 2331, pp. 613-621, 2002.

(C) Springer-Verlag Berlin Heidelberg 2002 


\section{Nonlinear Friction Observer}

It is very important to deal with uncertainty for the system speed and position in the mobile robot. Nonlinear friction causes stick-slip in the very low speed movement and is the original nonlinear factor, which makes tracking error in the path tracking control process due to stiction (breakaway force) and Stribeck effect [3]. In this paper we design the observer to estimate and compensate the friction torque in the 2-wheel mobile robot movement. Using the observer, it is possible to prevent the robot from breakaway and the navigation performance is improved.

\subsection{Nonlinear Friction Torque Modeling}

The friction with uncertain characteristic is changed by the speed and lubrication condition. The friction modeling [6][7] is described in equation (1).

$$
T_{f}(\omega(t))=T_{v}(\omega(t))+T_{c} \operatorname{sgn}(\omega(t))+T_{e}
$$

$T_{c}$ is coulomb friction torque. Normally the moving speed is proportional to the friction torque and the positive slope characteristic of the relation between friction torque and speed is shown in region B of Figure 1. Consequently, the friction torque is getting bigger in the very low speed scope. As it moves faster, the friction torque is getting smaller and this effect is called "Stribeck effect". Region A of Figure 1 shows the negative slope characteristic of the relation between friction torque and speed. $T_{v}$ is nonlinear viscous friction caused by stiction (breakaway force), which appears when the mobile robot starts. $T_{e}$ is static friction caused by Stribeck effect in the low speed scope and this negative characteristic describes the exponential decrement [4][5]. In Fig. 1, the friction torque modeling type is shown by the characteristic curve between the friction torque and moving speed. In this curve, the friction curve has the positive slope or the negative slope as the speed is changed. And the friction direction is changed with the opposite sign symmetrically as the sign of the speed is changed [6][7].

$$
\begin{gathered}
T_{f}(\omega(t))=\operatorname{sgn}(\omega) K\left(\alpha_{1}|\omega|+\beta_{1}+C_{1} e^{-\left(|\omega| / C_{2}\right)^{C_{3}}}\right) \\
\text { Where, }\left\{\begin{array}{l}
\operatorname{sgn}(\omega)=1 \text { at } \omega(t)>0 \\
\operatorname{sgn}(\omega)=-1 \text { at } \omega(t)<0
\end{array}\right.
\end{gathered}
$$

In equation (2), the parameter $\alpha_{1}$ is the torque coefficient of the viscous friction at region $\mathrm{B}$ and $\beta_{1}$ is the coulomb friction torque coefficient when the robot starts. $C_{1}$, $C_{2}$ and $C_{3}$ are the negative friction torque coefficients caused by Stribeck effect at region $\mathrm{A}$. $K$ is the gain coefficient for the entire friction. The friction torque function included in system is a nonlinear function of the speed. Depending on the motor direction, the function type is decided and the performance on the speed control is affected. Especially when the low speed and the high-speed navigation are repeated or when the forward and backward navigation are repeated, the control signal and the 


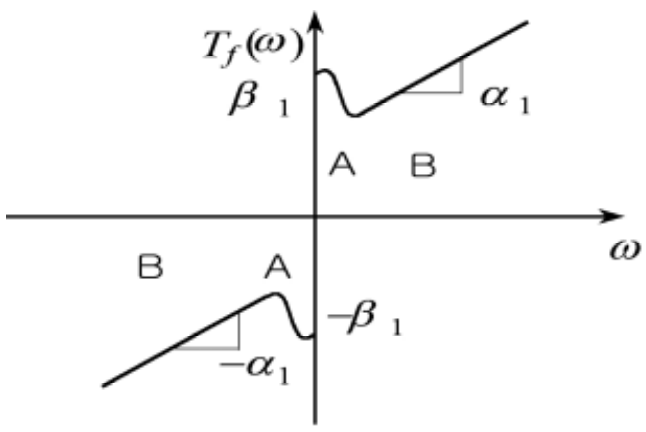

Fig. 1. Model of friction torque

navigation performance are disturbed. The friction torque is also shown as the disturbance that has a bad effect on the system control. In this paper, we consider the relationship between the tracking performance and breakaway in the planned path when the friction torques of the two wheels become unbalanced by stick and slip which are caused by inertia. When the robot turns left in the curve path after the straight movement, the speed of the right wheel increases and the speed of the left wheel decreases. In that case, the right wheel would keep the straight movement by inertia and the friction torque works to the contrary. Thus, stick that restrains the moving speed for the command of the torque increment happens. On the contrary, slip that would increase the speed for the decrement command happens.

\subsection{Friction Torque Observer}

The dynamic equation of the mechanical system is shown in equation (3).

$$
T=J \frac{d \omega}{d t}+\tau_{f}
$$

After converting equation (3) into Laplace Transformation, we get the equation (4).

$$
s J \omega_{M}=\tau_{M}-\tau_{f}
$$

Where, $J$ is the moment inertia of a system, $\tau_{M}$ is the motor torque, $\mathrm{s}$ is the Laplace operation. Assuming that the friction torque $\tau_{f}$ consists of the viscous friction torque and the coulombs friction in equation (4), equation (5) is obtained.

$$
\left\{\begin{array}{l}
s J \omega_{M}=\tau_{M}-\left(B \omega_{M}+\tau_{f c}\right) \\
\tau_{f_{c}}=\operatorname{sgn}\left(\omega_{M}\right) T_{f_{c}} \\
\operatorname{sgn}\left(\omega_{M}\right)=1 \quad\left(\omega_{M}>0\right) \\
\operatorname{sgn}\left(\omega_{M}\right)=0 \quad\left(\omega_{M}<0\right)
\end{array}\right.
$$


Where, $B$ is the friction coefficient $[\mathrm{Nm} /(\mathrm{rad} / \mathrm{s})]$. From equation (5), let $d \tau_{f c} / d t=0$ and $\hat{T}_{f c}$ is estimated as following equation (6).

$$
\hat{T}_{f c}=\frac{1}{\operatorname{sgn}\left(\omega_{M}\right)} \frac{\tau_{M}-(s J+B) \omega_{M}}{1+s \frac{J}{\operatorname{sgn}\left(\omega_{M}\right) L}}
$$

Where, $L$ is used as the free coefficient of the observer. In the denominator, the coefficient of s is a time constant as the first delay factor and we denote this as $K_{\tau}$.

$$
K_{\tau}=\frac{J}{\operatorname{sgn}\left(\omega_{M}\right) L}
$$

The sign of $K_{\tau}$ depends on the change of $\operatorname{sgn}\left(\omega_{M}\right)$. For the observer to be stable, it is necessary that the roots of characteristic equation are at the left half plane in the splane and choose $L$ to make the condition $K_{\tau}>0$. The feed forward compensated torque is decided as following equation (8).

$$
\hat{\tau}_{f c}=\operatorname{sgn}\left(\omega_{M}\right) \hat{T}_{f c}
$$

The speed control block using the proposed friction torque observer is shown in Fig 2. Where, $\mathrm{Q}(s)$ is defined in equation (9) and the motor torque $\tau_{M}$ is calculated in equation (10).

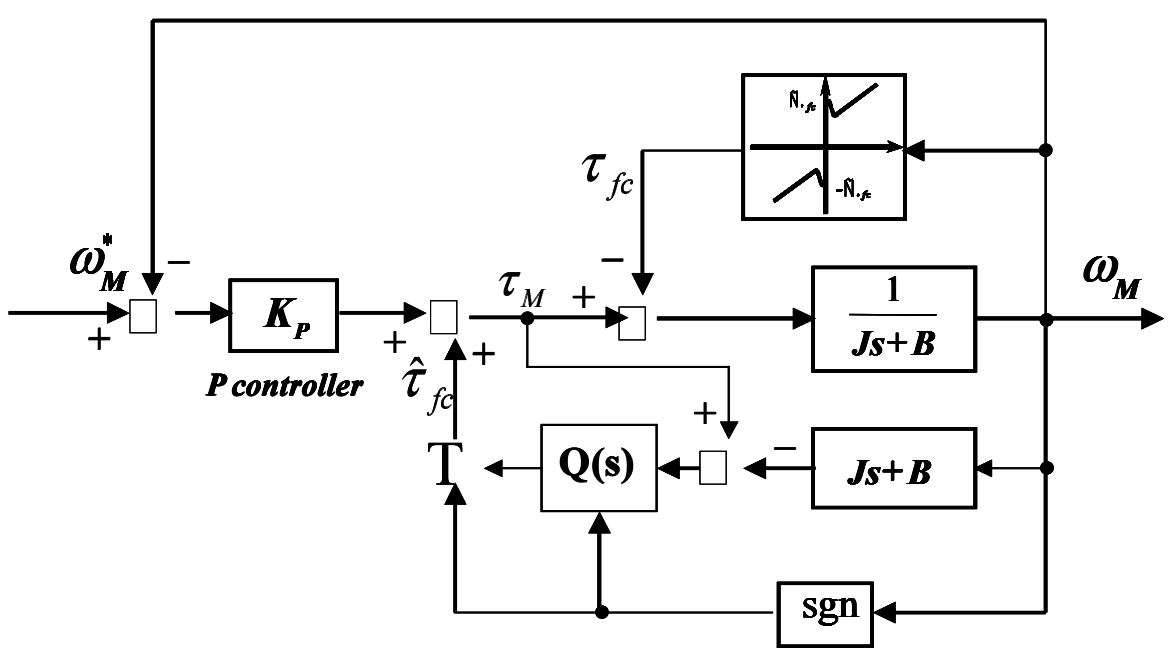

Fig. 2. The proposed friction torque observer 


$$
\begin{array}{r}
Q(s)=\frac{1}{\operatorname{sgn}\left(\omega_{M}\right)} \frac{1}{1+s \frac{1}{\operatorname{sgn}\left(\omega_{M}\right) L}} \\
\left.\tau_{M}=K_{p}{\left(\omega^{*}\right.}^{*}-\omega_{M}\right)+\hat{\tau}_{f c}
\end{array}
$$

Where, $K_{p}$ is the proportional speed gain and $\omega_{M}$ is the speed command. The proposed friction torque observer is a kind of the adaptive control and the free coefficient of the observer $L$ is changed as the sign of the moving speed is changed.

\section{Fuzzy Control System Modeling}

The performance of fuzzy controller is related to fuzzification, rule base, and $\mathrm{I} / \mathrm{O}$ linguistic parameters. Especially, premise and consequent linguistic values are very important factors in designing the fuzzy controller. And rule bases are decided after a lot of trials and errors. First of all, the direction error $e_{\theta}$ that is the angle between the robot's proceeding direction and the reference path and contour error $e_{c}$ that is the distance of the perpendicular line from the robot's weight center to the reference path are defined as the premise parameter for path tracking control [8]. And the consequent parameters $v_{d}$ and $v_{s}$ are defined in equation (11).

$$
\begin{aligned}
& v_{d}=v_{R}-v_{L} \\
& v_{s}=\frac{v_{L}+v_{R}}{2}
\end{aligned}
$$

Where, $v_{L}$ and $v_{R}$ are the left and right speed input values respectively. For the fuzzification method, the triangular membership functions are used.

Table 1. Fuzzy rule tables for the nonlinear friction observer

\begin{tabular}{|r|c|c|c|c|c|}
\hline$v_{d}$ & \multicolumn{5}{|c|}{$e_{\theta}$} \\
\hline $\mathrm{NL}$ & $\mathrm{PL}$ & $\mathrm{PL}$ & $\mathrm{PM}$ & $\mathrm{Z}$ & $\mathrm{NS}$ \\
\cline { 2 - 6 } $\mathrm{NS}$ & $\mathrm{PL}$ & $\mathrm{PM}$ & $\mathrm{PS}$ & $\mathrm{NS}$ & $\mathrm{NM}$ \\
\cline { 2 - 6 } $\boldsymbol{e}_{c} \mathrm{Z}$ & $\mathrm{PM}$ & $\mathrm{PS}$ & $\mathrm{Z}$ & $\mathrm{NS}$ & $\mathrm{NM}$ \\
\cline { 2 - 6 } $\mathrm{PS}$ & $\mathrm{PM}$ & $\mathrm{PS}$ & $\mathrm{NS}$ & $\mathrm{NM}$ & $\mathrm{NL}$ \\
\cline { 2 - 6 } $\mathrm{PL}$ & $\mathrm{PS}$ & $\mathrm{Z}$ & $\mathrm{NM}$ & $\mathrm{NL}$ & $\mathrm{NL}$ \\
\hline
\end{tabular}

\begin{tabular}{|r|c|c|c|c|c|}
\hline$v_{s}$ & \multicolumn{5}{|c|}{$e_{\theta}$} \\
$\mathrm{NL}$ & $\mathrm{M}$ & $\mathrm{S}$ & $\mathrm{M}$ & $\mathrm{M}$ & $\mathrm{Z}$ \\
\cline { 2 - 6 } $\mathrm{NS}$ & $\mathrm{S}$ & $\mathrm{S}$ & $\mathrm{M}$ & $\mathrm{M}$ & $\mathrm{S}$ \\
\cline { 2 - 6 }$e_{c} \mathrm{Z}$ & $\mathrm{S}$ & $\mathrm{S}$ & $\mathrm{L}$ & $\mathrm{S}$ & $\mathrm{S}$ \\
\cline { 2 - 6 } $\mathrm{PS}$ & $\mathrm{S}$ & $\mathrm{M}$ & $\mathrm{M}$ & $\mathrm{S}$ & $\mathrm{S}$ \\
\cline { 2 - 6 } $\mathrm{PL}$ & $\mathrm{Z}$ & $\mathrm{M}$ & $\mathrm{M}$ & $\mathrm{S}$ & $\mathrm{M}$ \\
\hline
\end{tabular}



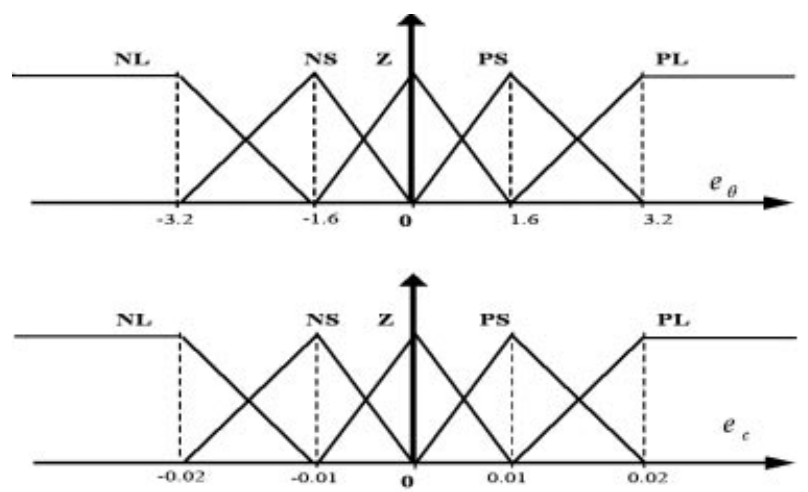

Fig. 3. Premise variable $e_{\theta}[\mathrm{deg}]$ and $e[\mathrm{~m}]$
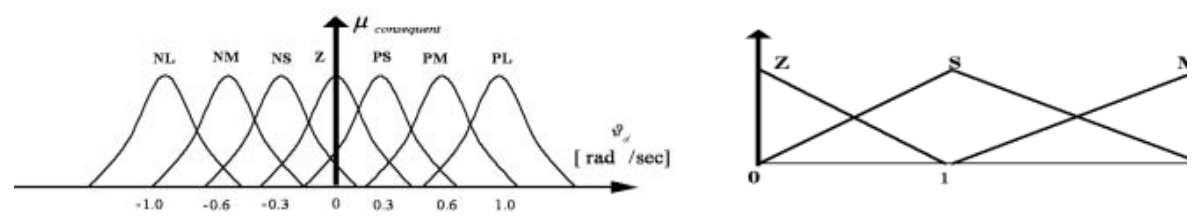

Fig. 4. Consequent variable $v_{d}[\mathrm{rad} / \mathrm{sec}]$ and $v_{s}[\mathrm{rad} / \mathrm{sec}]$

Each fuzzy rule base for the nonlinear friction observer consists of 25 rules that are shown in table1. These values in premise variables are decided with considering the distance among sensors. To apply the designed controller, we should make the membership function of premise variables and consequent variables. They are shown in Fig. 3 and Fig. 4. For the direction angle and the moving speed, each rule base is made. That is, even if the inputs are the direction error and the contour error, the outputs are the direction angle and the moving speed with two $2 \subseteq 1$ structures. For the fuzzy inference, Mamdani's product implication is used, and as t-norm and s-norm operations, algebraic product and max are used respectively. We use a lookup table to reduce calculation amount and time. After the sensor information is checked with this lookup table the control output is generated.

\section{Simulation}

In this paper, path tracking is simulated using Matlab program. We give the reference 

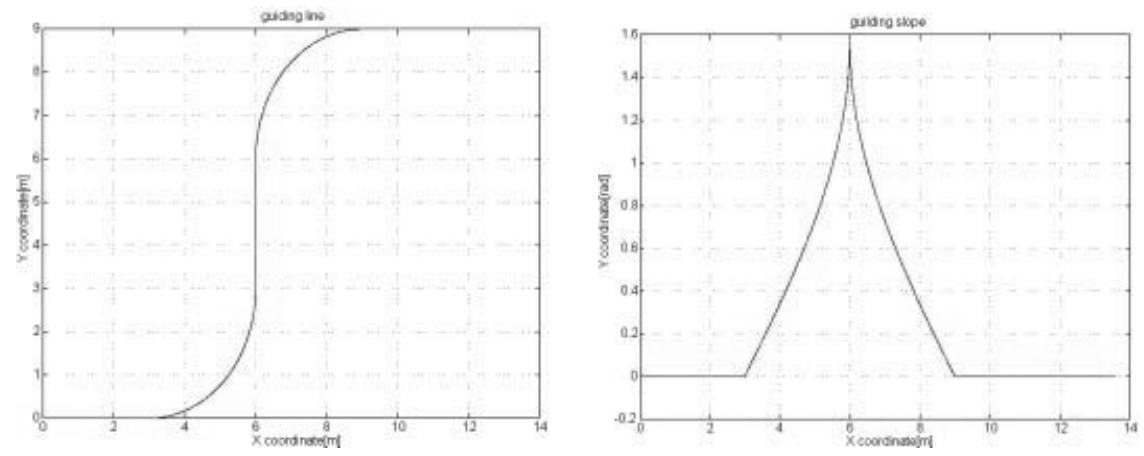

Fig. 5. The left figure shows the reference trajectory and the tangent slope of the trajectory is shown in the left figure

path and make the mobile robot follow that path. And the reference path is given like Fig. 5. We initialize the start position $(0,0)$ and assume that the initial direction is the same as the moving direction.

\subsection{Path Tracking without Observer}
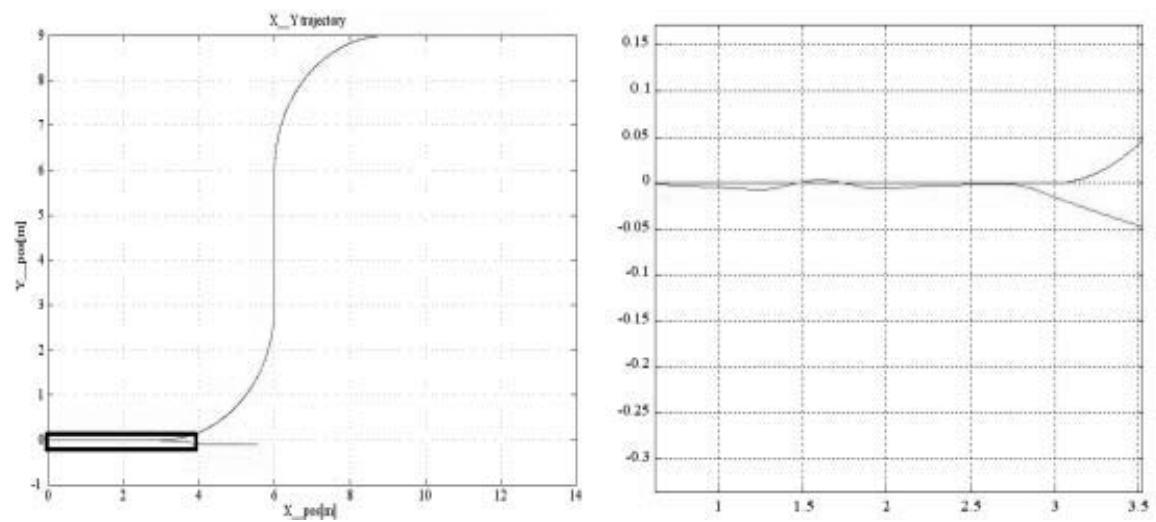

Fig. 6. The left result shows that the robot is out of the reference without using the observer and the right graph is a magnified one

The simulation result in Fig. 6 shows that when there is no difference between the left wheel inertia and the right wheel inertia or when the robot moves in the straight routines, it trace very well without the observer. But if there is some difference, the robot is out of the way as shown in Fig. 6 when it traces curves because the heavy slip effect caused by the nonlinear friction on the right wheel disturbs control commands. 

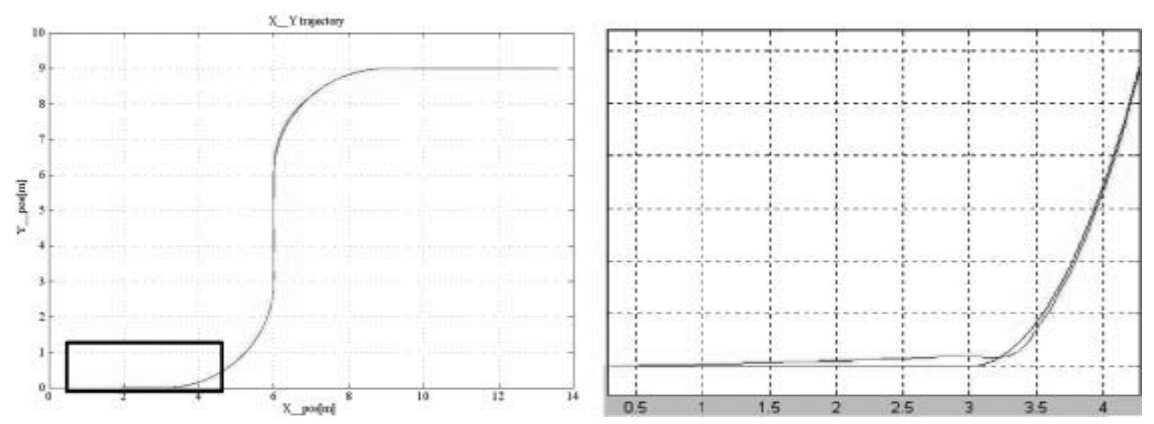

Fig. 7. The left result shows that the robot tracks the reference very well using the observer and the right graph is a magnified one

\subsection{Path Tracking with Observer}

In Fig. 7, the robot tracks both the straight path and the curve well. Especially when the robot follows the curve, the observer estimates the friction torque even though there is some difference between the left and the right wheel inertia. After that, the controller compensates the error caused by the nonlinear friction properly.

\section{Conclusions}

We have dealt with the servo controller to prevent from breakaway and to improve the path-tracking performance using the nonlinear friction observer. If stick or slip effect occurs in the driving parts, the friction torques of wheels become unbalanced and the torque command is disturbed. As a result, the path tracking performance gets worse and path-tracking failure happens. In this paper, the nonlinear friction observer is proposed to solve this problem. By consisting of this kind of a compensator, the disturbance caused by the nonlinear friction is compensated. That is, the main disturbance is compensated for and the input torque of the motor follows the command value of the direction angle well. Also, if the free parameter L, the first delay factor, is chosen properly, the system could be robust enough for a rapid command value change. In the fuzzy system, proper premise and consequent center values are decided by experimental knowledge and simulation. The system, therefore, would become stable and could have enhanced performance.

\section{References}

1. Sungchul Jee, "Fuzzy logic controls for CNC machine tools", a dissertation submitted in partial fulfillment of the requirements for the degree of Doctor of Philosophy in the University of Michigan, pp. 101-111

2. Kevin M. Passino, Stephen Yurkovich, "Fuzzy Control", Addison-Wesley, 1998.

3. B. Armstrong-Helouvry, P. Dupont, and C.C. de Wit, "A survey of models, analysis 
tools and compensation methods for the control of machines with friction." Automatica, vol. 30, no 7, pp. 1083-1138, 1994

4. C. C. de Wit, H. Olsson, K. J. Astorm, and P. Lischinsky. "The new model for control of systems with friction." IEEE Trans. Automat. Contr., vol. 40, pp. 419425, Mar. 1995.

5. M. Iwasaki and N. Matsui, "Observer-based nonlinear friction compensation in servo drive system." in Proc., IEEE 4th Int. Workshop AMC. 1996, pp. 344-348.

6. Makoto Iwasaki, "Disturbance-Observer-Based Nonlinear Friction Compensation in Table Drive System." IEEE/ASME Trans. on Mechatronics. vol. 4, No.1, March 1999.

7. P. E. Dupont, "Avoiding Stick-Slip through PD Control." IEEE Trans. on Automatic Control, vol. 39, no .5, pp. 1094 -1096, May. 1994.

8. L.Feng, "Cross-Coupling Motion Controller for Mobile Robots" IEEE Control Systems Magazine, Volume: 13 Issue: 6, pp. 35 -43 Dec. 1993. 